\title{
Albumin-producing hepatocytes derived from non- parenchymal liver cells of F344 rats in analbuminemic rat livers
}

Xia Yan ${ }^{1}$, Mitsuhiro Inagaki ${ }^{1}$, Jun Arikura ${ }^{1}$, Shinichi Kasai ${ }^{1}$

${ }^{1}$ Departments of Surgery, Asahikawa Medical College, 2-1-1-1, Midorigaoka-Higashi, Asahikawa 078-8510, Japan

Precious studies have shown that liver nonparenchymal cells (LNPCs) may contain hematopoietic stem cells. The present study was undertaken to investigate whether LNPCs can habor in the bone marrow cells (BMCs) of the F344alb irradated recipients can further raise hepatocytes in the liver after transplantation.

Materials and Methods. Male Fischer 344 rats (F344) were used as donors, and Male F344 congenic Nagase's analbuminemic rats (F344alb) as recipients. All F344 alb were divided into 4 groups: group I $(n=5)$ were untreated; group II $(n=5)$ were no transplantation, but had irradiation; group III $(n=5)$ were transplanted with $1 \times 10^{7}$ BMCs after irradiation; group IV $(n=5)$ were transplanted with $1 \times 10^{7}$ LNPCs after irradiation. The LNPCs prepared from the donors were infused via the penial veins of recipients immediatly after whole body irradiation (7.5 Gy). Animals were sacrificed at the 4th week after transplantation. Slices of the liver had albumin immunostaining with anti-rat albumin antibody.

Results. (1) Suvival rate: irradiated F344 alb had a mean $80 \%$ survival rate when they were transplanted with LNPCs (group IV), at meantime all irradiated rats without BMCs or LNPCs transplantation (group II) only had $\mathbf{2 0 \%}$ and those with BMCs transplantation had a $100 \%$ (group III) survival rate, respectively. (2) Although single or double cells of albumin positive (alb+) hepatocytes were seen in the livers of untreated F344alb (group I), clusters consisting of more than 6 alb+ hepatocytes were only detected in the livers of recipients that received transplantation of BMCs and LNPCs after irradiation (group III and IV).

Conclusions. These findings indicated that LNPCs can hobor in the BMCs of the F344alb recipients irradiated and further more can raise albumin-producing hepatocytes in the liver.

Cell Research (2008) 18:s166. doi: 10.1038/cr.2008.256; published online 4 August 2008 